\title{
OBJECT-CORE ORIENTED DATA MODELLING FOR TRACKING OF BEHAVIORS OF URBAN HEAT ISLANDS
}

\author{
R. Zhu ${ }^{1,2}$, E. Guilbert ${ }^{3}$, M.S. Wong ${ }^{4}{ }^{*}$ \\ ${ }^{1}$ Senseable City Laboratory, Singapore-MIT Alliance for Research and Technology, Singapore - rui.zhu@ smart.mit.edu \\ ${ }^{2}$ Dept. of Land Surveying and Geo-informatics, The Hong Kong Polytechnic University, Hong Kong, China - r.zhu@ connect.polyu.hk \\ ${ }^{3}$ Dept. of Geomatics Sciences, Laval University, Québec, Canada - eric.guilbert@ scg.ulaval.ca \\ ${ }^{4}$ Dept. of Land Surveying and Geo-informatics, The Hong Kong Polytechnic University, Hong Kong, China - 1s.charles@polyu.edu.hk
}

\section{Commission IV, WG IV/1}

KEY WORDS: Spatiotemporal data modeling; Urban heat islands; Database Management System; Geographical Information Science; Remote sensing

\begin{abstract}
:
Modeling thematic and spatial dynamic behaviors of Urban Heat Islands (UHIs) over time is crucial to understand the evolution of this phenomenon and the city micro-climate. Previous studies conceptualized that a UHI can only have a single life period with spatial behaviors (i.e. areal changes and topological transformations). However, a UHI can also appear and disappear periodically several times expressed by thematic and spatial integrated behaviors, which has not been established yet. Thus, this study conceptualizes each UHI as an object which has thematic and spatial behaviors simultaneously and proposes several graphs to depict periodic life-time transitions triggered by the behaviors. The model was implemented in an object-relational database, and air temperatures collected from a number of weather stations were interpolated as temperature images each hour for six weeks. Results indicated that the model could track the spatial and thematic evolution of UHIs through continuous time effectively, and also revealed the periodical patterns and abnormal cases of UHIs over a city.
\end{abstract}

\section{INTRODUCTION}

Urban Heat Island (UHI) is one of the problems that arouse great attention from the public, which is an environmental phenomenon where temperatures in urban areas are higher than its surrounding rural areas (Nichol et al. 2009). It is reported that annual temperature difference between urban and rural areas is normally between 1 and 3 degrees Celsius, and it can be reached as high as 12 degrees Celsius in the evening (Unite States Environmental Protection Agency 2017). Dynamic behaviors of UHIs continuously occur in space over time (Buyantuyev and Wu 2010, Kourtidis et al. 2015, Wu, et al. 2012). There is a need of designing an effective model to describe its dynamic behaviors and track its evolutionary trends integrating with space and time. Spatiotemporal data modeling, containing but not limited to three classes (i.e. field-based data modeling, object-based data modeling, and event-based data modeling) (Bothwell and Yuan 2010, Goodchild et al. 2007, Miller and Bridwell 2009), has been conducted in several research disciplines, which provides an enlightening approach to develop an adaptive data model for tracking the UHI phenomenon.

Object-based data models have been studied to present dynamic geographical phenomena. The objective of these models is to describe the spatiotemporal behavior of objects observed in sequences of images. Geographical phenomena can be defined as field objects corresponding to geo-objects with an internal structure defined by variations of field-like properties within the boundary of the object (Goodchild et al. 2007). Their dynamics is driven by their activities, events and processes, and can be observed through changes and movements (Yuan and Hornsby 2008).

\footnotetext{
${ }^{*}$ Corresponding author
}

Such an approach works especially well with environmental phenomena such as UHI. The reason is that all the discrete spatial properties can be associated with a time series relationship so that the relationship can be linked to the corresponding objects.

Following this, an object shall contain information of space and it is identifiable, relevant of interest, and describable through designed characteristics as stated in (Mattos et al. 1993). More specifically, an object can be described by static properties, structural characteristics, and behavior characteristics (Worboys 1994). The object-oriented data model normally requires the integration of specific disciplinary knowledge for different applications such as remote sensing theories for image classification (Chen et al. 2012), computer science technologies for 3D data visualization (Shi et al. 2003), and GIS methods for traveling behavior disaggregation (Frihida et al. 2002). However, all these do not provide methods for the association and areal change between fields. Since UHI is a typical field phenomenon, a comprehensive modeling method which combines both field and object data modeling shall be considered.

Topological relationships are defined by topological transitions of two objects in two consecutive time steps. Spatiotemporal topological process involves single and several entities, which establishes three different categories to describe evolution: evolution of an individual object, functional relationships between several objects, and evolution of spatial structures between several objects (Claramunt and Thériault 1995). In a more detailed level, evolution of a single object contains three types of processes: (i) basic process for the representation of attribute variation while without spatial changes; (ii) transformation processes focusing on the changes of shape and size; (iii) and movement processes involving locational movement. Furthermore, functional relationships involve replacement processes which describe a sequence 
of objects that construct one process or occupy the same location in space, and diffusion processes which transfer characteristics between two or more objects.

Further research considered topological relationships by modeling splitting and merging behavior of spatiotemporal objects in two ways, i.e., splitting may result in the continuous existence of the original object or the replacement of new ones, and merging may lead to the continuous existence of one of the original objects or the replacement of a new one (Nixon and Hornsby 2010). However, other topological transformation operations for spatiotemporal field-object with single attribute, such appearance of a new object versus disappearance of an existing one that may be used for describing behavior of a UHI, were not systemically modeled. Another study explicitly described these transformations (Del Mondo et al. 2013), which presents spatiotemporal relationships in a graph to continuously track changes of objects over time. Other transitions that affect the shape and thematic attribute of a field-object were established in two graphs (Zhu et al. 2016). As a consequence, a graph to systematically describe locational movements has not been built yet.

Object-oriented feature-based models can not only present topological relations among geometric elements but also incorporate semantic relations among features (Tang et al. 1996). In the study, a geographical feature is constructed by feature objects, each of which also contains a geometric object. Associations between feature objects are built by semantic relations. Essentially, all these abstraction mechanisms can be applied for abstracting useful information from UHIs that share a similar or the same characteristics in different granularities whenever all their behaviors can be tracked through designed groups. For example, all the UHIs which cluster together in a high density and expand significantly during a short time period can be extracted to draw a hotspot representing a serious UHI. In this consideration, a more appropriate definition of UHI will be proposed in this study.

This study aims to provide a new object-oriented model where UHIs are viewed as spatiotemporal objects with internal structures which are able to determine their relationships through time and space. The purpose of the new model is to describe spatiotemporal processes of UHIs more reasonably so as to provide a more convicing description of the UHI behaviors. The model can be easily developed in a database management system that allows the recording of the behaviors in a time series and executing of pattern mining through simple queries.

The rest of this paper is organized as follows. Section 2 proposes a new defitinion for a UHI. Section 3 presents a new method for tacking behaviors of UHIs. Section 4 proposes a method for the visualization of the locational movement of UHIs. Section $5 \mathrm{em}-$ pirically evaluates the proposed method in a case study. Finally, Section 6 draws concludes and future work.

\section{UHI AS A FIELD-OBJECT}

A UHI can be conceptualized as a two-dimensional field where its areal extent can expand, contract, or remain stable possibly because temperatures warm up, cool down, or keep constant through continuous time and space. Therefore, a UHI can be seen as a variable field whose thematic attribute is the temperature measured in urban areas that are with certain degrees Celsius difference from a reference temperature observed in rural areas at the same time. Even though some studies indicated that UHI may be a localized phenomenon which does not shift from its original location and the displacement is not obvious (Hua and Wang 2012, Jalan and Sharma 2014), this study attempts to model its locational behavior to reveal various movement trends. Based on the above conceptualization, a UHI can be integrated as a field-object with four components:

- the first spatial component defined by a polygon delineating its extent;

- the second spatial component defined by a point to describe its location and construct its historical trajectory;

- a thematic component which is the temperature intensity observed from the thermal image and defined as a field variable; and

- a temporal component allowing the description of its behaviors in consecutive time instants.

On top of these, a UHI can go through spatiotemporal transformations describing a change of status or its relationships with other UHIs. For instance, a UHI may split into two UHIs or disappear when the temperature intensity decreases. This shows that topological transformation determined by thematic characters can also trigger the transition of its status.

For a UHI phenomenon, the changes of temperatures (i.e. the thematic property) determine the shape and location changes (i.e. the spatial property). Recently, UHI intensity has been widely defined as the temperature difference between urban and rural areas (Martin-Vide et al. 2015). This definition makes a UHI occur as long as its temperatures are higher than the reference rural temperature, and spatial extent of the UHI is deterministic. However, a UHI shall be at least with certain temperature higher than the reference rural temperature to distinguish the peaks of intensity and define several UHIs where there was only one before, which may create inclusions between UHIs. For example, one study has suggested that micro heat islands in the same urban area changed sensitively in short term, and had negative effects to the public health (Goggins et al. 2012), which indicates that the study has allowed several small UHIs to occur in a large urban area. In this consideration, a UHI can be viewed as an urban area where its internal temperatures are with a given temperature higher than its reference rural temperature. Thus, a UHI can be formulated.

- An urban heat island is a two-dimensional urban area where its internal temperatures are with a given temperature (i.e. the magnitude) higher than its reference rural temperature.

In a wide temporal scale, a UHI can disappear and appear periodically because of the fluctuation temperatures, acting as an object in different statuses. Thereby, a UHI can be defined in a more formal manner.

- An urban heat island is a field-object having a magnitude to represent the UHI intensity, a location to depict the geographic location, a zone to describe a variable extent, and a time to provide a temporal scale for the description of the thematic and spatial evolution. 


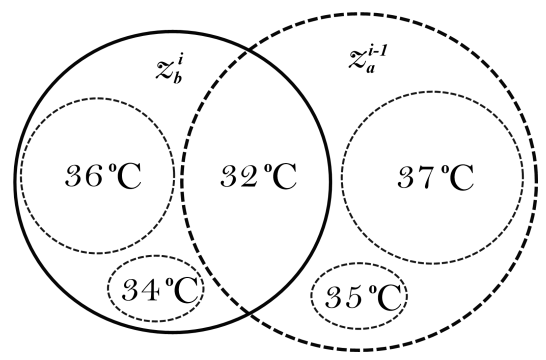

Figure 1. Two zones touch each other apparently but they have no association essentially since their areas equaling to the maximum temperature are not in the overlapping area.

\section{CORE-ORIENTED BEHAVIOR MODELING}

A UHI is caused by some heat released from a building, the traffic or whatsoever and this heat diffuses from this source. The UHI is the area over which the temperature increases because of this heat. The source can be a point, a line (e.g. vehicles along a road) or an area (e.g. a whole building). Thus, given a UHI created by a heat source, the UHI is static if the source is static while it is dynamic if the source is moving.

Air flows make thermal exchanges of the air and lead to variation of air temperatures. Because of this reason, some temperatures within a certain region may decrease continuously, which can cause that the original hot region divides into two parts with fuzzy boundaries, and finally splits into two independent less-hot regions. In an opposite scenario, two regions can expand continuously and get closer with each other because the heat in the two regions are accumulating. At certain time instant, the two regions may interact with each other with thermal exchanges and start fusion processing immediately, creating a new fuzzy boundary. Thus this could be a complicate and dynamic process, either two regions merge together and become a larger region or a large region neutralizes a smaller one but remains the same. In this reasoning, zones of UHIs can have area changes and topological transformations simulatenously, and distribution of ambient temperatures in the UHI extent can also vary significantly that determine its behaviors ultimately.

A previous study proposed a set of filiations by investigating the relations between zones in two consecutive time instants (Zhu et al. 2016). For example, two zones that have significant overlapping in two continuous time instants can be viewed as the same UHI in two statuses. Hence, a thematic behavior is determined by comparing the temperatures, and a spatial behavior is assigned in comparison of the two areas. However, using this method to determine two zones as two consecutive statuses of the same object is not reliable if: (i) air temperatures in some areas of the zone are equal or higher than the temperature, (ii) these areas take significant proportion of the zone, but (iii) these areas are not located in the overlapping area of the two zones. In this case, it is more convincing to suggest that the two zones are overlapping with each other but substantially have no association with each other. Let $z_{n}^{i}$ be a zone index by $n$ at time instant $t_{i}$ in a spatial and temporal domain. As it is drawn in Figure 1, the maximum temperature of the zone is used as the representative temperature so that areas having the the maximum temperatures of 37 degrees Celsius at $t_{i-1}$ and 36 degrees Celsius at $t_{i}$ are separated without any association.

A better way is to consider spatial distribution of the representative temperature to model spatial filiations between zones. A

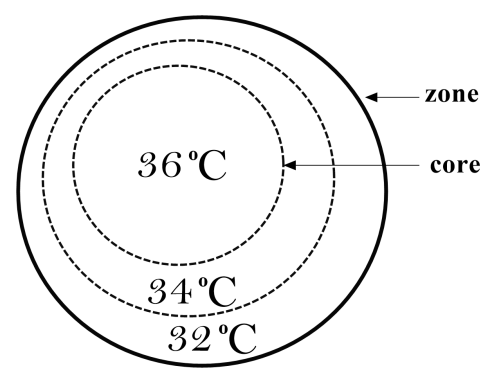

Figure 2. Core is inside of the zone, which represents an extent of a set of the spatial contiguous of the statistical temperatures.

study proposed a core region to describe complex landform of canyons, which is one of the most representative characteristics for canyons (Guilbert and Moulin 2017). With the similar approach, a zone can have a representative core region where absolute temperatures within the core region are equal or higher than the representative temperature. As drawn in Figure 2, a core is an enclosed region in the zone that temperatures within the region equal to the representative temperature (i.e. the maximum temperature) of 36 degrees Celsius. For the ease of representation, a new concept of core is introduced so that the core-oriented behavior modeling can be proposed.

- A core is an internal area of the zone, which contains a complete set of the spatial contiguous of temperatures that are equal or higher than the representative temperature.

\subsection{Spatial behavior of the UHI}

Zones of $\left(z^{i-1}, z^{i}\right)$ that have significant overlapping were perceived as the same UHI, and hence specific filiation can be further identified (Zhu et al. 2016). However, thematic cores of both zones may not locate in the overlapping region but are separated in the non-overlapping region of each zone. Then, it is reasonable to say that they are only apparently overlapping and they are not associated to the same UHI. For the other example, zones of $\left(z^{i-1}, z^{i}\right)$ may overlap with each other insignificantly while their cores are all located in the overlapping region. In this case, it is convincing to state that these two zones belong to the same object, having locational shifting. Therefore, spatial relationships between zones and spatial distribution of thematic temperatures shall be considered simultaneously to model filiations between zones.

When several UHIs interact with each other in the same spatiotemporal domain (i.e. one city can have multiple UHIs simultaneously), it is crucial to firstly determine the relationship between zones at two consecutive time instants to model their dynamic behaviors, i.e., to list the sequences of zones in a temporal domain that belong to the same UHI. According to the above the perception, relationship between zones are essentially determined by topological relationships of cores between different zones. Therefore, at least one core $r_{n}^{i-1}$ associating with the other one $r_{n}^{i}$ is the pre-condition to build zone filiations. For a pair of spatially associated zones $\left(z_{n}^{i-1}, z_{n}^{i}\right)$, zone filiations in view of area changes are thus organized as:

- expansion when $r_{n}^{i-1}$ associates with $r_{n}^{i}$ and geometry expands from $z_{n}^{i-1}$ to $z_{n}^{i}$;

- continuation when $r_{n}^{i-1}$ associates with $r_{n}^{i}$ and spatial extent of geometry is similar from $z_{n}^{i-1}$ to $z_{n}^{i}$; and 
- contraction when $r_{n}^{i-1}$ associates with $r_{n}^{i}$ and geometry contracts from $z_{n}^{i-1}$ to $z_{n}^{i}$.

At a time instant, UHIs alternatively have transformation or areal change (Zhu et al. 2016). However, this study suggests that both can happen simultaneously in two specific scenarios, i.e., separation and annexation when the zone still exists. The reason is that deformation of the original zone $z_{n}^{i-1}$ in these two affiliations is not interrupted even at a specific time instant when topological transformation occurs such that area changes still can be determined as usual. For pairs of zones $\left(\left\{z^{i-1}\right\},\left\{z^{i}\right\}\right)$, topological transformations are refined as:

- splitting when one zone $z_{n}^{i-1}$ only spatially associates with two zones $z_{p}^{i}$ and $z_{q}^{i}$;

- separation when $r_{n}^{i-1}$ associates with $r_{n}^{i}$ and $z_{n}^{i-1}$ also has spatial association with $z_{n}^{i}$ and $z_{p}^{i}$

- merging when two zones $z_{p}^{i-1}$ and $z_{q}^{i-1}$ only spatially associate to one zone $z_{n}^{i}$; and

- annexation when $r_{n}^{i-1}$ associates with $r_{n}^{i}$ and two zones $z_{n}^{i-1}$ and $z_{p}^{i-1}$ are spatially associated to one zone $z_{n}^{i}$.

While, two special transformations may happen that either $z_{n}^{i-1}$ or $z_{n}^{i}$ cannot determine any associations, leading to the creation of a new zone or destruction of an existing zone:

- appearance when one zone $z_{n}^{i}$ has no association with any other zones at $t_{i-1}$; and

- disappearance when one zone $z_{n}^{i-1}$ is not related with any zones at $t_{i}$.

\subsection{Thematic behavior of the UHI}

The same as the zone-oriented approach, representative temperature is needed to describe evolution of the thematic property continuously for a long time period. In addition, four statistical values can be used as the indices, i.e., the maximum, mean, median, and mode values of a complete set of the thematic property values, all of which are able to reveal thematic characteristics in different aspects. Obviously, all the cores meet the same condition that temperatures in the cores are equal to or higher than the thematic temperature. When a pair of zones have been determined as the same object, thematic behaviors hence can be qualitatively described as increasing, stationary, and decreasing by comparing the representative temperatures $s_{n}^{i-1}$ and $s_{n}^{i}$, e.g. the maximum temperatures of the two zones.

Compared with the zone-oriented modeling, the core-oriented modeling describes a more constrained area (i.e. moving from a zone to one or multiple cores of the zone) for the spatial distributions of the statistical temperatures. Even there can be several cores in a single zone, their thematic statistics are homogeneous since they all meet the same statistical condition, which thus does not cause difficulties in the description of the thematic behaviors.

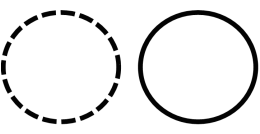

disjoint
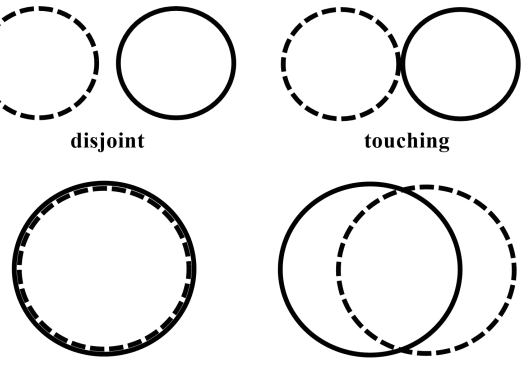

complete coincidence
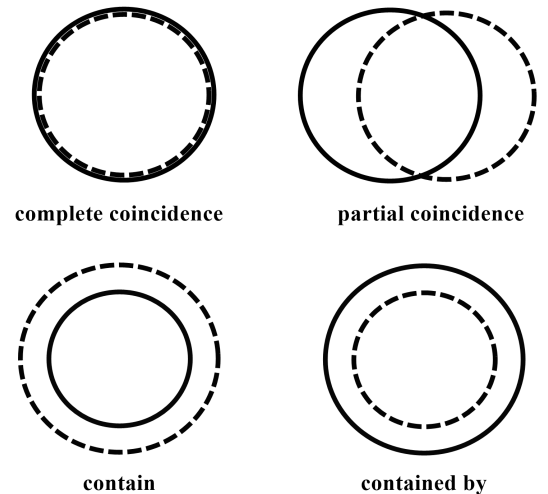

partial coincidence
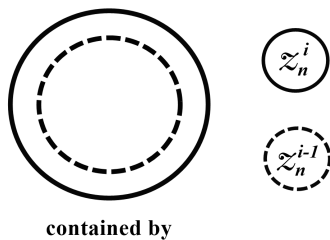

Figure 3. Spatial relationship between a pair of zones $\left(z_{n}^{i-1}, z_{n}^{i}\right)$.

\subsection{Locational behavior of the UHI}

It is widely acknowledged that movement of the objects will lead to the displacement of their locations. Thus, locational behaviors can be represented by different displacements of zones. An effective method to identify different displacements is to determine topological relationships between zones. For example, a static UHI without any movement means that their zones may coincide with each other, and a UHI significantly moves far away from its origin would correspond to the disjoint of two zones at $t_{i}$ and $t_{i+1}$. In this situation, several types of topological relationships between zones have to be refined and constructed.

Let $z_{n}^{i}$ denote the zone of a UHI $u_{n}(n \in\{1, \ldots, m\})$ at the time instant $t_{i}$. A study proposed a Voronoi-based nine-intersection (V9I) model which includes a topological relation between two areas with a four-intersection (area, area) matrix (Long and $\mathrm{Li}$ 2013). According to the needs of this study, spatial relationships between any two zones in two time instants of the same spatial domain are summarized in Figure 3 and reorganized as:

- disjoint when $z_{n}^{i-1}$ does not connect with $z_{n}^{i}$;

- touching when $z_{n}^{i-1}$ just contacts with $z_{n}^{i}$;

- complete coincidence when $z_{n}^{i-1}$ completely overlaps with $z_{n}^{i}$

- partial coincidence when $z_{n}^{i-1}$ partially overlaps with $z_{n}^{i}$;

- containing when $z_{n}^{i-1}$ includes $z_{n}^{i}$; and

- contained by when $z_{n}^{i-1}$ is inside of $z_{n}^{i}$.

Movement should mainly rely on the displacement of the core and should be done once the while history has been reconstructed. This study considers that a pair of zones $\left(z_{n}^{i-1}, z_{n}^{i}\right)$ have closer relations and have more iterative behaviors when they are spatially closer with each other. Zones which are stationary shall continuously stay at the same place without any locational displacement while extent variation, such as expansion, contraction, or stabilization, is allowed. A pair of zones can also have notable but insignificant locational displacement, allowing them to have locational changes within certain distance. The last possibility 


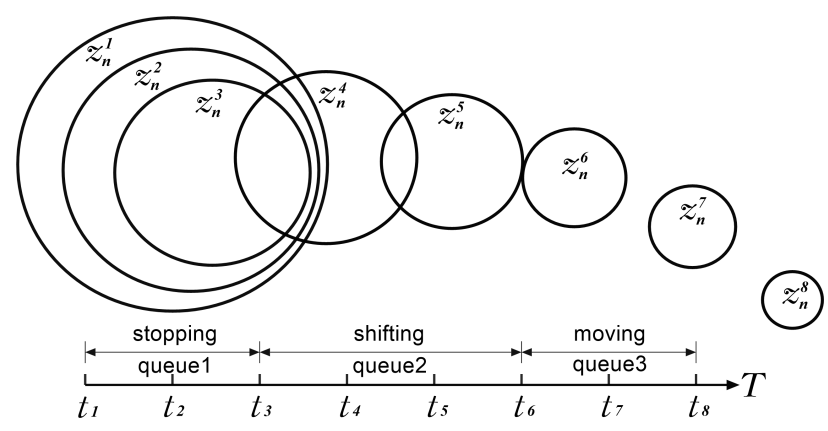

Figure 4. A UHI are continuously stopping, shifting, and moving in three consecutive queues from $t_{1}$ to $t_{8}$.

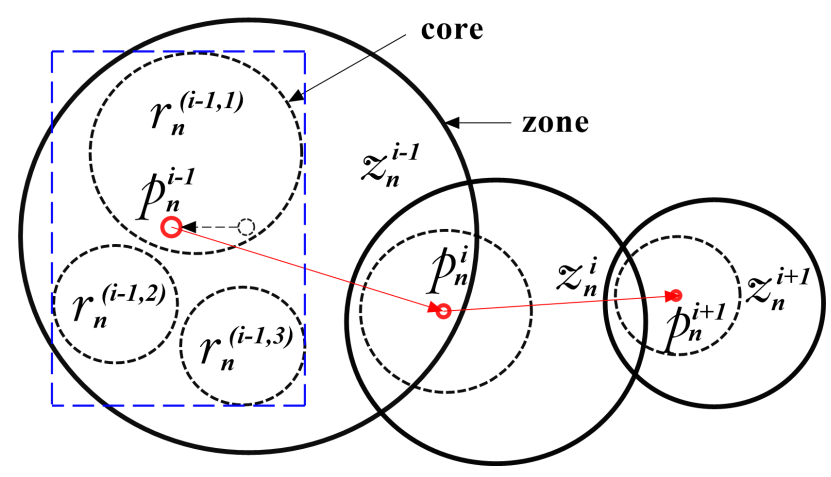

Figure 5. Shift the center of mass of the zone to the centroid of the minimum enclosing rectangle of all the cores.

is that zones maintaining their spatial extent are moving away dramatically from their origins. The two zones are thus conceptualized as:

- stopping when $\left(z_{n}^{i-1}, z_{n}^{i}\right)$ are containing, contained by, or complete coincidence;

- shifting when $\left(z_{n}^{i-1}, z_{n}^{i}\right)$ are touching, or partial coincidence; and

- moving when $\left(z_{n}^{i-1}, z_{n}^{i}\right)$ are disjoint.

An uninterrupted displacement corresponds to a queue. The displacement can be either static (i.e. stopping) or dynamic (i.e. shifting or moving). Since a UHI does not move in a regular rule, determining its consecutive queues would explicitly describe the displacement patterns of the locations. As drawn in Figure $4, z_{n}^{1}$ continuously contracts as $z_{n}^{2}$ and $z_{n}^{3}$, where extent of $z_{n}^{2}$ is covered by $z_{n}^{1}$ and $z_{n}^{3}$ is covered by $z_{n}^{2}$, which leads to a stopping queue for the locational behavior of a UHI. Hence, the UHI is shifting since zones partial coincide and touch with each other during $\left[t_{3}, t_{6}\right]$. Then, the UHI obtains a moving because their zones are disjoint. Notably, time periods for a sequence and a queue does not necessarily coincide with each other but also can be interlaced. For example, a UHI can have no displacement for a time period, which corresponds to a stopping queue. Simultaneously, areal extent of the UHI can continue and expand during the same time period, making two consecutive sequences. This interlacing shall be helpful to uncover different causative factors.

\section{TRAJECTORY DETERMINATION}

When all the behaviors are established, an auxiliary task is visualizing areal extents and trajectory of each UHI. Areal extents can be visualized by simply plotting the polygon of each zone $z_{n}^{i}$ but locations has to be wisely determined to construct historical trajectories. As mentioned above, there may be several cores that values of the thematic property in these cores equal to the thematic temperature. Let $k$ number of these cores in the boundary of $z_{n}^{i}$ be denoted by $\left\{r_{n}^{(i, k)}\right\}(k=\{1, \ldots, l\})$. Since cores essentially represent hotspots of the zone caused by spatial distribution of the interested temperatures, it is much more meaningful to consider that the centroid of the zone is determined by the set of cores instead of setting it at the center of the zone. Since there can be several cores that their areas are almost the same, still setting the centroid as the center of either core is not convinced. An appropriate solution is locating it at the centroid of the minimum enclosing rectangle of all the cores and noted by $p_{n}^{i-1}$ as shown in Figure 5. This solution considers the contribution of each core to the centroid displacement. As such, routing trajectory for each UHI can be depicted by a polyline noted as $\left\{p_{n}^{1}, p_{n}^{2}, \ldots, p_{n}^{i}\right\}$, and it can be easily extended by adding a new point $p_{n}^{i+1}$ at $t_{i+1}$ to the trajectory.

\section{LOCATIONAL-GRAPH BASED TRACKING}

To track the displacement of UHIs and to reveal evolutionary trends of the locational movements, a new graph is thus proposed as: $\mathbf{G}_{Q}=\left(\mathbf{Q}, \mathbf{F}_{q}\right)$ where $\mathbf{Q}$ denotes a time-series of locations and $\mathbf{F}_{q}$ represents a set of filiations between locations, which essentially relies on the existence of the thematic graph $\mathbf{G}_{C}$. Note $\mathbf{F}_{q}=\mathbf{Q}_{q} \cup \mathbf{L}_{q}$ so that $\left(q_{n}^{i}, q_{n}^{j}\right) \in \mathbf{Q}_{q}$ describes each pair of consecutive queues belonging to the same UHI, which is viewed as an edge of the graph to offer qualitative descriptions of the locations, and $\left(q_{n}^{i}, q_{m}^{j}\right) \in \mathbf{L}_{q}$ indicates that one or several queues of different objects $q_{m}^{j}$ associate with the same queue $q_{n}^{i}$ of the UHI $u_{n}$. Since a queue contains a set of centroids for the same UHI over $\left[t_{i}, t_{j}\right]$ that ultimately constructs one particular type of the locational filiation (e.g. stopping, shifting, or moving), a queue thus can be more precisely noted as $q_{n}=\left\{l_{n}^{i}, \ldots, l_{n}^{j}\right\}$ which satisfies $\forall k, i<k \leq j,\left(l_{n}^{k-1}, l_{n}^{k}\right)=\left(l_{n}^{i}, l_{n}^{i+1}\right) \in \mathbf{Q}_{q}$.

Apart from two transitions for sequences and chains that have been proposed (Zhu et al. 2016), a new transition to present trends of locations is gathered. Let $q_{n}^{i_{j-1}}, q_{n}^{i_{j}}$ and $q_{n}^{i_{j+1}}$ denote three consecutive queues of the same UHI $u_{n}$ that transitions connect every two of them in a temporal domain. Compared with the thematic property which has quantitative records for the intensity, locational movement does not include a magnitude description domain such that the transitions have neither peaks nor troughs. Associating with the two edges $\left(q_{n}^{i_{j-1}}, q_{n}^{i_{j}}\right)$ and $\left(q_{n}^{i_{j}}, q_{n}^{i_{j+1}}\right)$, transitions can be redefined:

- if $q_{n}^{i_{j-1}}$ is stopping, $q_{n}^{i_{j}}$ is shifting, and $q_{n}^{i_{j+1}}$ is moving, then $u_{n}$ obtains an accelerating;

- if $q_{n}^{i_{j-1}}$ has a movement, $q_{n}^{i_{j}}$ shifts, and $q_{n}^{i_{j+1}}$ stops, then $u_{n}$ reaches a decelerating;

- if $q_{n}^{i_{j}}$ either moves or shifts while $q_{n}^{i_{j-1}}$ and $q_{n}^{i_{j+1}}$ are stopping, then the queue $q_{n}^{i_{j}}$ is viewed as an active phrase. $u_{n}$ consequentially reaches and leaves an activation; and 


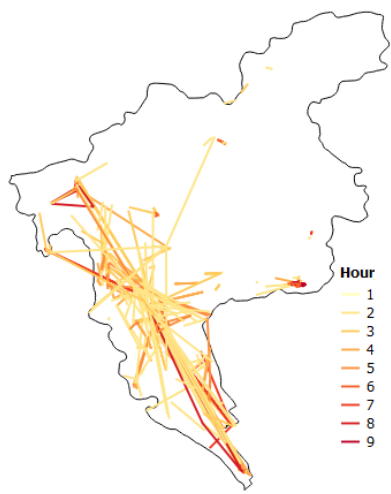

(a) Traj. in 07/31-08/06

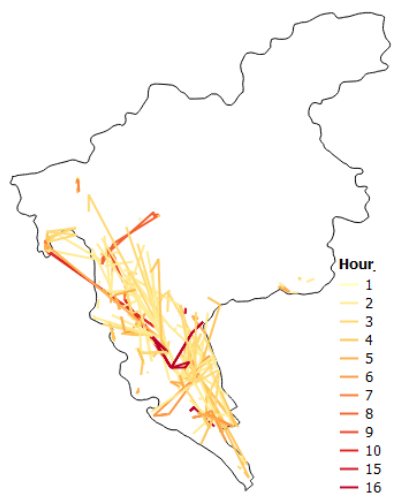

(c) Traj. in 09/25-10/01

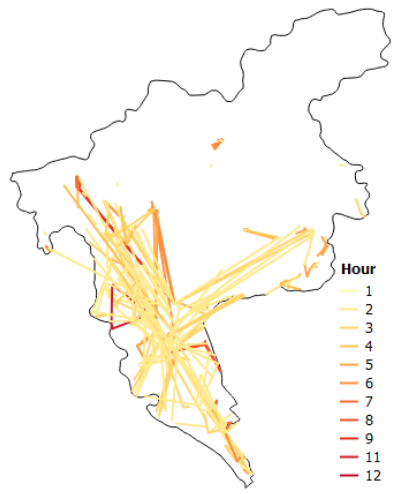

(e) Traj. in 11/20-11/26

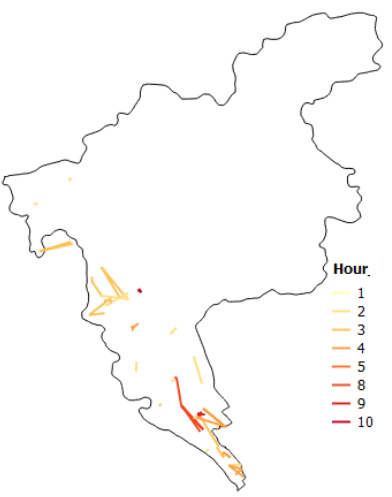

(b) Traj. in 07/31-08/06

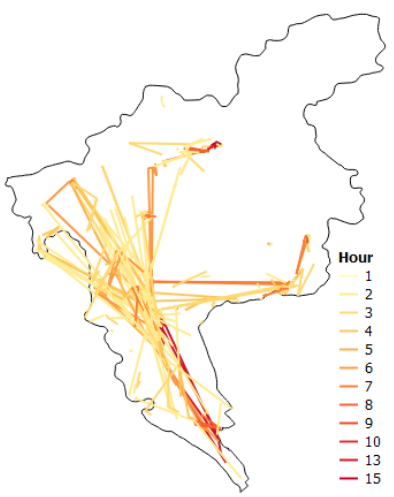

(d) Traj. in 10/23-10/29

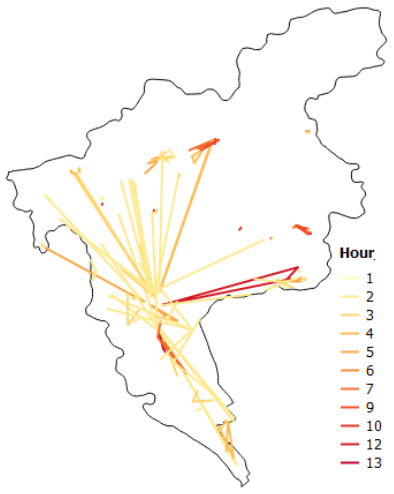

(f) Traj. in 12/18-12/24

Figure 6. Trajectories of UHIs ( $m=3$ ) for six weeks. Longer life-span of a UHI is presented in darker color.

- if $q_{n}^{i_{j}}$ stops while $q_{n}^{i_{j-1}}$ and $q_{n}^{i_{j+1}}$ either move or shift, then the queue $q_{n}^{i_{j}}$ is conceptualized as a quiescence. $u_{n}$ reaches quiescence and resumption during the two transitions.

\section{EXPLORATORY DISCOVERY}

Guangzhou in China is selected to evaluate the effectiveness of the model, where UHI in this city is evident since temperature difference can reach up to 13 degree Celsius (Chen et al. 2016). Near-surface air temperatures were collected by 216 automatic weather stations every hour for six weeks (i.e., July 31 - August 6, July 28 - September 3, September 25 - October 1, October 23 - October 29, November 20 - November 26, and December 18 December 24) in year 2015, covering a continual of six months.

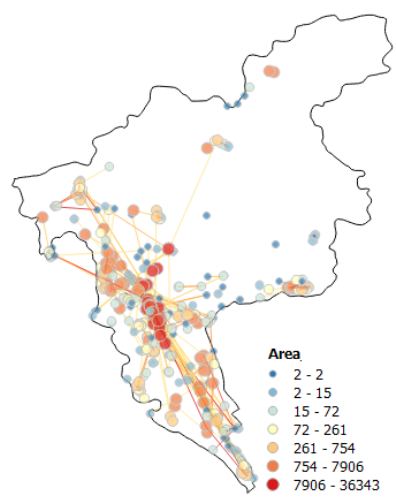

(a) Zone area in 07/31-08/06

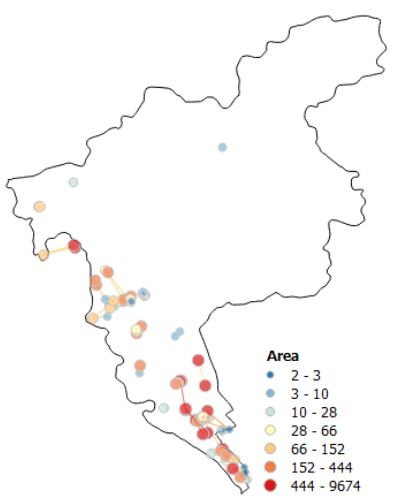

(b) Zone area in $07 / 31-08 / 06$

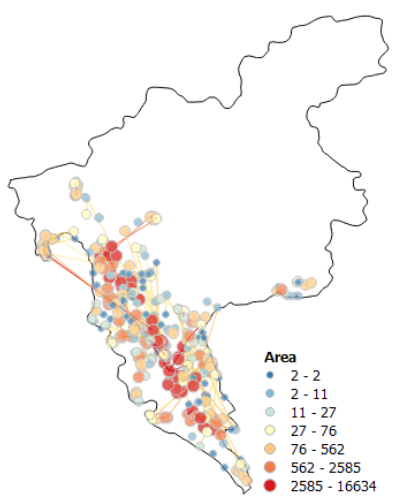

(c) Zone area in 09/25-10/01

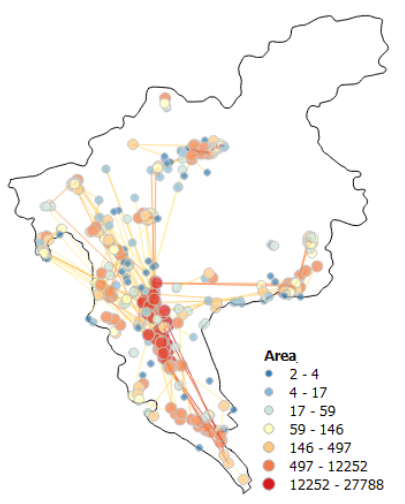

(d) Zone area in $10 / 23-10 / 29$

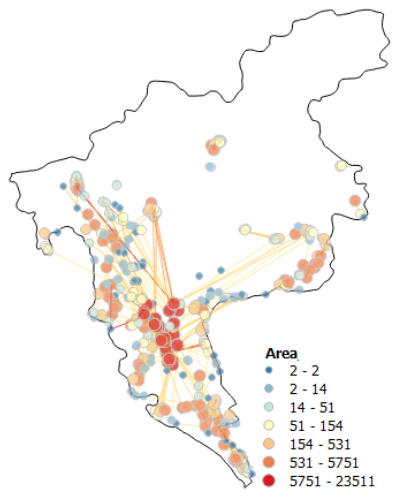

(e) Zone area in $11 / 20-11 / 26$

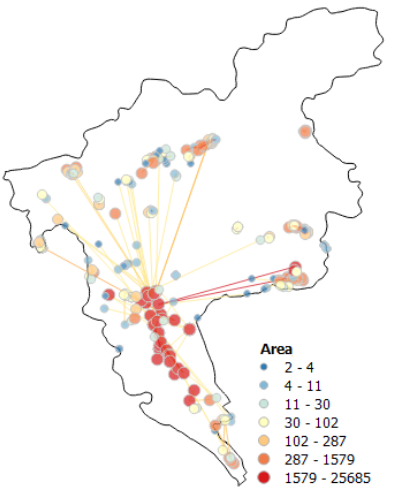

(f) Zone area in $12 / 18-12 / 24$
Figure 7. Zone of UHIs associated with their trajectories. Lager area of the zone has larger circle size.

Thereby, a series of near-surface thermal images are generated by interpolating hourly data, and are used as the input data of the model. Ordinary Kriging was used since it models the surface by assuming that an overriding trend exists in the data sets, which is beneficial for highlighting the hotspot character of UHIs (Chai et al. 2011, Hofstra et al. 2008, Irmak et al. 2010, Stahl et al. 2006). Temperatures observed at the rural area were used as the reference temperature to extract zones of UHIs, which is not only located in the rural area (i.e. land cover is forest) but also next to the urban areas of Guangzhou.

The proposed model is interactive and allows SQL query to construct trajectories of UHIs and summarize their active hours. Figure 6 draws all the trajectories of UHIs over six independent weeks. The figure shows that UHIs associate in the core urban area of Guangzhou and shift back and forth to urban areas of the 


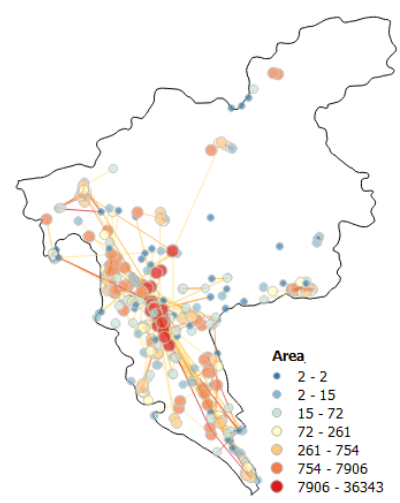

(a) Zone areas when $m=3$

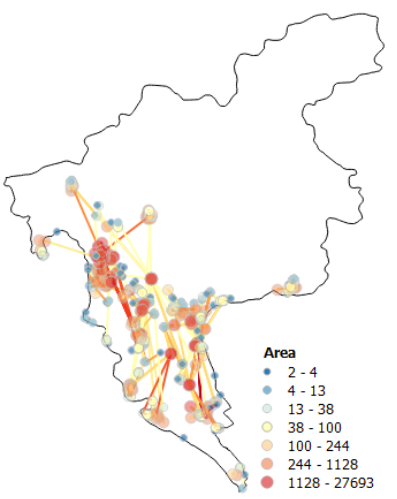

(b) Zone areas when $m=4$

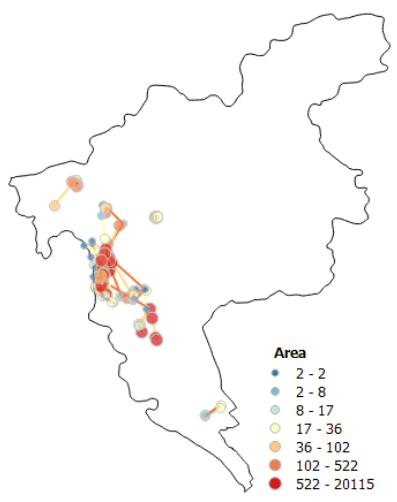

(c) Zone areas when $m=5$

Figure 8. Zone areas of UHIs in different magnitudes associate with their trajectories between July 31 and August 6.

surrounding districts periodically when the magnitude is intermediate $(m=3)$. Accordingly, a series of zones of each UHI categorized by area are plotted along with the trajectories (Figure 7). It obviously shows that larger zones are clustering in the core urban area while smaller zones are discretized in different districts, apart from raining days (Figure 7(b)). The two figures also reveal that: (i) the UHI phenomenon becomes inconspicuous from summer to winter since trajectories are getting sparse; (ii) UHIs having the same magnitude maintain their travels between cores and suburbs disregarding with the change of seasons; and (iii) their extents contract moving from cores to suburbs while the extents expand when turning round.

The above findings motivate us to further investigate trajectories when UHIs are in higher magnitudes. Focusing on a single week between July 31 and August 6, areal information of zones and their occurrence time along with the trajectories are drawn in Figure 8 and Figure 9 respectively. It is found that trajectories are getting sparser and shorter, and gradually centralized in the core urban area with the increase of magnitudes. This means that UHIs with higher intensities would have smaller extents and tend to associate in more density urban areas. Figure 9 also visualizes temporal occurrence of UHIs. It presents that UHIs with low magnitude $(m=3)$ can exist all through the day covering a large area, while UHIs with higher magnitude $(m=5)$ overwhelmingly occur in the night occupying much smaller area. All these findings suggest that UHIs having a low magnitude would associate in several urban areas while their occurrence time can decrease over seasons. In contrast, UHIs with a high magnitude would associate in a specific urban area while their occurrence time can only be in the night.
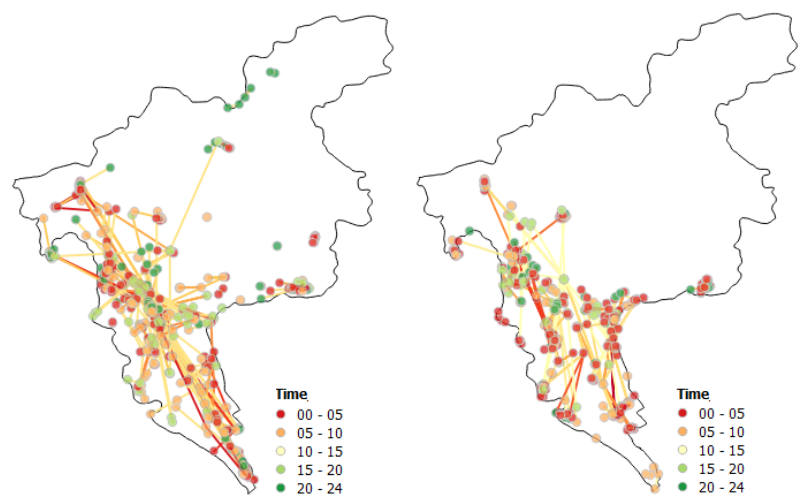

(a) Time of zones when $m=3$

(b) Time of zones when $m=4$

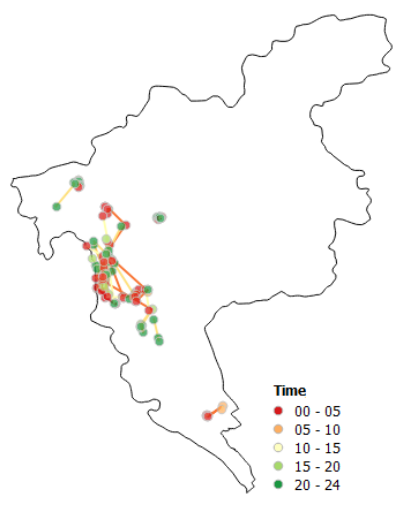

(c) Time of zones when $m=5$

Figure 9. Time-based location of zones associated with their trajectories between July 31 and August 6.

\section{DISCUSSION AND CONCLUSION}

The proposed model allows geographical phenomenon to be conceptualized as a field-object with interal core strctures such that its evolution can be modeled as continuous of the dynamic behaviors, acting as an object either develops independently or interacts with other objects with topological transformations. Empirical evaluation based on a developed database system suggests that the model is effective to record the changes of UHIs and is able to reveal evolutionary trends of UHIs. UHIs with higher magnitudes are more locationally associated while variability of their trajectories maintains in all the seasons.

In each set of the experiment, a specific magnitude was used to extract zones of UHIs and temperatures within the zones are at least with a magnitude difference from the referenced rural temperatures. However, location of the rural temperatures used for extracting the extent of UHIs was selected empirically, which could not represent all the rural temperatures to precisely extract the boundaries of UHIs. An approach to determine the referenced rural temperatures more accurately is creating an outer-buffer of the investigated urban areas with a certain width so that the buffer shall cover certain rural areas. Hence, a threshold temperature can be determined by averaging all the rural temperatures within this buffer zone. The advantage of this approach is that all the rural temperatures are taken into account so that uncertainty of the reference rural temperatures caused by selection of the site can be avoided.

The current model can be used to investigate UHI at a finer scale of urban areas such that underlying mechanism of the UHIs phe- 
nomenon may be revealed by synchronously tracking other hypothetical influential factors. For instance, heat produced by vehicular flows can be a major contribution to the UHI in some specific places where the heat also has the peaks during the rushing hours in the morning and afternoon. In consideration of the urban morphology, different wind directions can either accumulate or disperse the heat when the wind travels through different wind corridors in a city. Future work can track the UHI evolution using the developed model and simulate the urban dynamics (e.g. vehicles and winds) with multi-disciplinary knowledge, and analyze their spatio-temporal correlations systematically to explore and understand potential factors.

\section{REFERENCES}

Buyantuyev, A., Wu, J., 2010. Urban heat islands and landscape heterogeneity: linking spatiotemporal variations in surface temperatures to land-cover and socioeconomic patterns. Landscape Ecology, 25, pp. 17-33.

Bothwell, J., Yuan, M., 2010. Apply concepts of fluid kinematics to represent continuous space-time fields in temporal GIS. Annals of GIS, 16(1), pp. 27-41.

Claramunt C., Thériault M., 1995. Managing Time in GIS: An Event-Oriented Approach. Proceedings of the International Workshop on Temporal Databases: Recent Advances in Temporal Databases, pp. 23-42.

Chai, H. X., Cheng, W. M., Zhou, C. Q., Chen, X., et al., 2011. Analysis and comparison of spatial interpolation methods for temperature data in Xinjiang Uygur Autonomous Region, China. Natural Science, 3(12), pp. 999-1010.

Chen G., Hay, G. J., Carvalho, L. M. T., Wulder, M. A., 2012. Object-based change detection. International Journal of Remote Sensing, 33(14), pp. 4434-4457.

Chen, G., Zhao, L., Mochida, A., 2016. Urban Heat Island Simulations in Guangzhou, China, Using the Coupled WRF/UCM Model with a Land Use Map Extracted from Remote Sensing Data. Sustainability, 8(7), 628. doi:10.3390/su8070628

Del Mondo, G., Rodríguez, M. A., Claramunt, C., Bravo, L., et al., 2013. Modelling Consistency of Spatio-temporal Graphs. Data \& Knowledge Engineering, Elsevier, 84(1), pp. 59-80.

Frihida, A., Marceau, D. J., Thériault, M., 2002. Spatio-Temporal Object-Oriented Data Model for Disaggregate Travel Behavior. Transactions in GIS, 6(3), pp. 277-294.

Goodchild, M. F., Yuan, M., Cova, T. J., 2007. Towards a general theory of geographic representation in GIS. International Journal of Geographical Information Science, 21(3), pp. 239-260.

Goggins, W. B., Chan, E. Y. Y., Ng, E., Ren, C., et al., 2012. Effect Modification of the Association between Short-term Meteorological Factors and Mortality by Urban Heat Islands in Hong Kong. PLoS One, 7(6), e38551.

Guilbert, G., Moulin, B., 2017. Towards a Common Framework for the Identification of Landforms on Terrain Models. ISPRS International Journal of Geo-Information, 6(12), pp. 1-21.

Hua, L., Wang, M., 2012. Temporal and spatial characteristics of urban heat island of an estuary city, China. Journal of Computers, 7(12), pp. 3082-3087.

Hofstra, N., Haylock, M., New, M., Jones, P., et al., 2008. Comparison of six methods for the interpolation of daily, European climate data. Journal of Geographical Research, 113, D21110. doi:10.1029/2008JD010100
Irmak, A., Ranade, P. K., Marx, D., Irmak, S., et al., 2010. Spatial Interpolation of climate variables in Nebraska. Transactions of the ASABE, 53(6), pp. 1759-1771.

Jalan, S., Sharma, K., 2014. Spatio-temporal asscessment of land use / land cover dynamics and urban heat island of Jaipur city using satellite data. The International Archives of the Photogrammetry, Remote Sensing and Spatial Information Sciences, XL-8, pp. 767-772.

Kourtidis, K., Georgoulias, A. K., Rapsomanikis, S., Amiridis, V., et al., 2015. A study of the hourly variability of the urban heat island effect in the Greater Athens Area during summer. Science of the Total Environment, 517, pp. 162-177.

Long, Z. Li, S., 2013. A complete classification of spatial relations using the Voronoi-based nine-intersection model. International Journal of Geographical Information Science, 27(10), pp. 2006-2025.

Miller, H. J., Bridwell, S. A., 2009. A Field-Based Theory for Time Geography. Annals of the Association of American Geographers, 99(1), pp. 49-75.

Mattos, M. M., Meyer-Wegener, K., Mitschang, B., 1993. Grand tour of concepts for object-orientation from a database point of view. Data and Knowledge Engineering, 9, pp. 321-352.

Martin-Vide, J., Sarricolea, P., Moreno-García1, M. C., 2015. On the definition of urban heat island intensity: the "rural" reference. Frontiers in Earth Science, 3, pp. 24.

Nixon, V., Hornsby, K. S., 2010. Using geolifespans to model dynamic geographic domains. International Journal of Geographical Information Science, 24(9), pp. 1289-1308.

Nichol, J. E., Wing, Y. F., Lam, K., Wong, M. S., 2009. Urban heat island diagnosis using ASTER satellite images and 'in situ' air temperature. Atmospheric Research, 94, pp. 276-284.

Stahl, K., Moore, R. D., Floyer, J. A., Asplin, M. G., et al., 2006. Comparison of approaches for spatial interpolation of daily air temperature in a large region with complex topography and highly variable station density. Agricultural and Forest Meteorology, 139, pp. 224-236.

Shi, W. Z., Yang, B. S., Li, Q. Q., 2003. An object-oriented data model for complex objects in three-dimensional geographical information. International Journal of Geographical Information Science, 17(5), pp. 411-430.

Tang, A. Y., Adams, T. M., Usery, E. L., 1996. A spatial data model design for feature-based geographical information systems. International Journal of Geographical Information Systems, 10(5), pp. 643-659.

Unite States Environmental Protection Agency, 2017. Heat Island Effect. Retrieved from https://www.epa.gov/ heat-islands

Wu, F., Wang, X., Cai, Y., Yang, Z., et al., 2012. Spatiotemporal analysis of temperature-variation patterns under climate change in the upper reach of Mekong River basin. Science of the Total Environment, 427-428, pp. 208-218.

Worboys, M., 1994. Object-oriented approaches to georeferenced information. International Journal of Geographical Information Systems, 8(4), pp. 385-399.

Yuan, M., Hornsby, K., 2008. Computation and Visualization for Understanding Dynamics in Geographic Domains. CRC Press.

Zhu, R., Guilbert, E., Wong, M. S., 2016. Object-oriented tracking of the dynamic behavior of urban heat islands. International Journal of Geographical Information Science, 31(2), pp. 405424. 